\title{
A Boosting-Based Approach to Refine the Segmentation of Masses in Mammography
}

\author{
Mario Molinara, Claudio Marrocco, and Francesco Tortorella \\ Dept. of Electrical and Information Engineering \\ Università degli Studi di Cassino e del Lazio Meridionale \\ via Di Biasio, 43 Cassino (FR), Italy \\ \{m.molinara, c.marrocco, tortorella\}@unicas.it
}

\begin{abstract}
In this paper we present an algorithm for finding an accurate estimate of the contour of masses in mammograms. We assume that a rough estimate of the region containing the mass is known: in particular it is available the location of an area inside the mass (core) and a closed curve beyond which the mass does not extend. The proposed method employs a boosting-based classifier trained on the core and on a background region beyond the external contour, so that it provides an accurate estimate of the mass contour by classifying unlabeled pixels between the core and the external contour. The proposed approach is useful not only for automatic localization of mass contour, but also as a powerful tool during annotation of mammograms, given that an user provides interactively an estimate for the core and the external contour of the mass. The approach has been verified on a set of mammograms showing very encouraging results.
\end{abstract}

Keywords: Mammography, Mass Segmentation, Boosting.

\section{Introduction}

Breast cancer is one of the most common cause of cancer deaths among women [1]. At present, early diagnosis represents the only way to successfully attack the disease with a suitable therapy and to significantly improve the chance of survival for patients with breast cancer. For this reason programs of wide mass screening for the female population at risk have been carried out in many countries. In such programs, mammography plays a central role since it makes it possible to detect abnormalities such as calcifications and masses as well as subtle signs such as architectural distortion and bilateral asymmetry [10] using low doses of radiation. In recent years there has been a great effort in research to develop computer-aided detection or diagnosis (CAD) systems that use computer technologies to assist radiologists in the analysis of mammographies [3|9 3 11 8 ]. These systems are particularly useful in mass screening where a high number of images must be examined by a radiologist. In fact, CAD systems can help reduce the number of errors (and therefore the social and economical costs) by increasing both the sensitivity and the specificity of the diagnosis. CAD can thus be seen as a diagnosis made by a radiologist who uses the output from computerized analysis of medical images as a "second opinion" in detecting lesions and making diagnostic decisions [5].

A. Petrosino (Ed.): ICIAP 2013, Part II, LNCS 8157, pp. 572-580, 2013.

(C) Springer-Verlag Berlin Heidelberg 2013 
In this framework, several methods have been proposed for automatic localization of masses on mammograms. Their approaches is typically made of two steps: the first one is the detection of the internal core of a mass which differs from normal breast tissue in gray-level intensity values or in local texture or morphological measures. The second step (segmentation) is devoted to determine the whole area of the mass, which should be extracted as precisely as possible. In fact, the area and the shape of the mass provides fundamental diagnostic information to be used in a subsequent classification phase.

For this purpose two main groups of methods are used: region-based segmentation and edge-based segmentation. In the first one, the characteristics of the pixels of the core are taken into account and similar neighboring pixels are included until no more pixels satisfy the criteria for inclusion in the area of the mass. In edge-based segmentation, the edges between the mass and the normal tissue are detected and linked together to form a closed contour. Unfortunately, both the approaches present some problems which affect the quality of the segmentation. The formulation of the stopping rule is the weak point in region-based segmentation: in several cases, the region growing process fails to stop at mass border and includes also part of the normal tissue. On the other hand, edge-based segmentation can miss some part of the actual contour since gradient is not sufficiently high: sometimes, in fact, the border between the mass and the normal tissue is given by a difference in texture more than a difference in pixel intensity.

In this paper we present an algorithm for finding an accurate estimate of the contour of masses in mammograms, partially inspired by the method proposed in [2]. We assume that a rough estimate of the region containing the mass is known: in particular, there should be available the location of the core of the mass (as required by the regionbased methods) and an external contour, i.e., a closed curve beyond which the mass does not extend. The proposed method employs a boosting-based classifier trained on the core and on a background region beyond the external contour, so that it provides an accurate estimate of the mass contour by classifying unlabeled pixels between the core and the external contour. In this way, the inclusion of pixels in the area of the mass is not driven by the similarity with the core, but is based on a discriminative model which robustly distinguish between actual mass and normal tissue. Incidentally, the proposed approach is useful not only as a refinement of the output of a segmentation step in automatic mass localization, but also as a powerful tool during annotation of mammograms, provided that an user supplies interactively an estimate for the core and the external contour of the mass.

\section{The Method}

Our approach consists in accurately estimating the contour of masses that can be present in a mammogram. The goal is both to help the radiologists in their work of manual detection of the mass contour and to improve the output of a CAD system for mass detection. For this purpose, in this paper, we consider as already detected the suspicious masses in the image and we focus on the refinement of the contour considering it as a two-class classification problem. In the following, we will refer to the pixels belonging to the mass as positive samples and to the pixels belonging to the background as negative. In this way, a dichotomizer can be trained on the labeled pixels of the mass 
and of the background and then applied to the unlabeled pixels of the border region. Our method is principally a pixel-based AdaBoost classifier. However, since to draw the contour we need to handle spatial interaction between neighboring pixels, the weak classifier and the feature vector of every pixel to be classified is modified as in [2] to capture local image statistics.

\subsection{Features}

First, since the proposed method is pixel-based, a feature set has to be evaluated for each pixel to be classified. Such features take into account both gray-level and spatial information. Features belonging to the first group are the value of the intensity of the considered pixel, the square of such value to better highlight color differences and the mean of the intensity values of the pixels belonging to a $n \times n$ window centered at the pixel. The other kind of features, instead, has to capture both intensity and spatial information and for this purpose we have referred to the Histogram of Oriented Gradients (HOG) descriptors, a variation of Scale Invariant Feature Transform (SIFT) descriptors [7] commonly used in the human detection framework [4]. The basic idea of such features is that local object appearance and shape are characterized by the distribution of local intensity gradients and edge directions. Thus, in our case building a HOG representation in the neighborhood of each pixel let us capture the edge and the gradient structure that characterizes the local shape of the mass.

In practice a HOG is implemented considering a $n \times n$ window centered at the pixel and evaluating, for each window, a local histogram of gradient directions over the pixels of the region. To this end, the first step is the computation of $x$ and $y$ derivatives that we have implemented by means of the Prewitt operator. Such operator used a $3 \times 3$ kernel as follows:

$$
G_{x}=\left[\begin{array}{ll}
-1 & 0+1 \\
-1 & 0+1 \\
-1 & 0+1
\end{array}\right] * I \quad G_{y}=\left[\begin{array}{rrr}
+1 & +1 & +1 \\
0 & 0 & 0 \\
-1 & -1 & -1
\end{array}\right] * I
$$

where $I$ is a region of interest (ROI) containing both the mass and a part of the background. Then, the angle of the gradient of each pixel in the ROI is evaluated and the 8-bin HOG in the neighborhood of each pixel is built. Finally, the entropy from the histogram $H O G$ relative to the pixel $p$ is calculated as:

$$
\operatorname{Entropy}(p)=-\sum_{i=1}^{8} H O G(i) \cdot \log _{2} H O G(i) \text {. }
$$

\subsection{Classifier}

Once each pixel has been associated to a feature vector the classifier can be trained. As previously stated our approach relies on a reformulation of AdaBoost suitably modified to model the spatial interaction between pixels. Let us consider the feature vector and the labels of a collection of $N$ pixels: $\left(\mathbf{x}_{i}, y_{i}\right), \forall i=1, \ldots, N$. AdaBoost consists of 
a boosting-based ensemble of weak classifiers $h_{t}(\mathbf{x})$ added in subsequent rounds $t=$ $1, \ldots, T$ and linearly combined by weights $\alpha_{t}$ to build the strong classifier:

$$
H(\mathbf{x})=\sum_{t=1}^{T} \alpha_{t} h_{t}(\mathbf{x})
$$

Every weak classifier $h_{t}(\mathbf{x})$ can be trained on positive and negative samples generating dichotomizers working on the considered features but not considering any spatial interaction between neighboring pixels. To this end, it is possible to train a different weak classifier that takes in input the current label of the neighboring pixels where with "current label" we refer to the output of the classifier at each round of AdaBoost. This means that after each boosting iteration in addition to the feature vector of each pixel, it is also useful to evaluate the predicted labels of its neighbors that are used to train a new weak classifier. Thus, in each round of AdaBoost we have to train two weak classifiers: the first, the data weak, is trained on each pixel independently while the second, the spatial weak, is trained on the predicted label of neighborhoods of pixels. However, only one of the two weak classifiers is considered for the strong classification system since, in each round, AdaBoost chooses the weak classifier that minimizes the classification error. As a result, the strong classifier is still a weighted sum of weak classifiers, be it data or spatial classifiers, where the weights $\alpha_{t}$ at each round are chosen equal to:

$$
\alpha_{t}=\frac{1}{2} \ln \left(\frac{1-\epsilon_{t}}{\epsilon_{t}}\right),
$$

with $\epsilon_{t}$ indicating the error of the data or the spatial weak classifiers. A detailed version of the training algorithm is reported in Algorithm 1 .

We have actually employed least squares dichotomizers for both spatial and data classifiers. Let us consider $d$ as the number of features, every weak classifiers $h(\mathbf{x})$ : $\mathcal{R}^{d} \rightarrow\{-1,+1\}$ is defined as:

$$
h(\mathbf{x})=\mathbf{v}^{T} \mathbf{x} .
$$

where $\mathbf{v} \in \mathcal{R}^{d}$ is a separating hyperplane computed through the weighted linear square regression. Let us consider the matrix $\mathbf{A}$ where the rows $\mathbf{A}_{i}$ correspond to the feature vector of a pixel $\mathbf{x}_{i}$ augmented with the constant 1 (i.e., $\mathbf{A}_{i}=\left[\mathbf{x}_{i} 1\right]$ ) and let us indicate with $\mathbf{W}$ the diagonal matrix of the weights $\mathbf{w}_{i}$ associated to each pixel. Then, the weighted sum-squared error criterion is given by:

$$
J=\|\mathbf{W}(\mathbf{A} \mathbf{v}-\mathbf{y})\|^{2} .
$$

The solution for $\mathbf{v}$ minimizing $J$ is:

$$
\mathbf{v}=(\mathbf{W A})^{\dagger} \mathbf{W} \mathbf{y}
$$

where (WA $)^{\dagger}$ is the pseudo-inverse of WA. If (WA $)^{T}$ WA is nonsingular, then an approximate solution for $\mathbf{v}$, commonly employed in the least squares minimization problem, is obtained through:

$$
\mathbf{v}=\left(\mathbf{A}^{T} \mathbf{W}^{T} \mathbf{W A}\right)^{-1} \mathbf{A}^{T} \mathbf{W}^{T} \mathbf{W} \mathbf{y}
$$




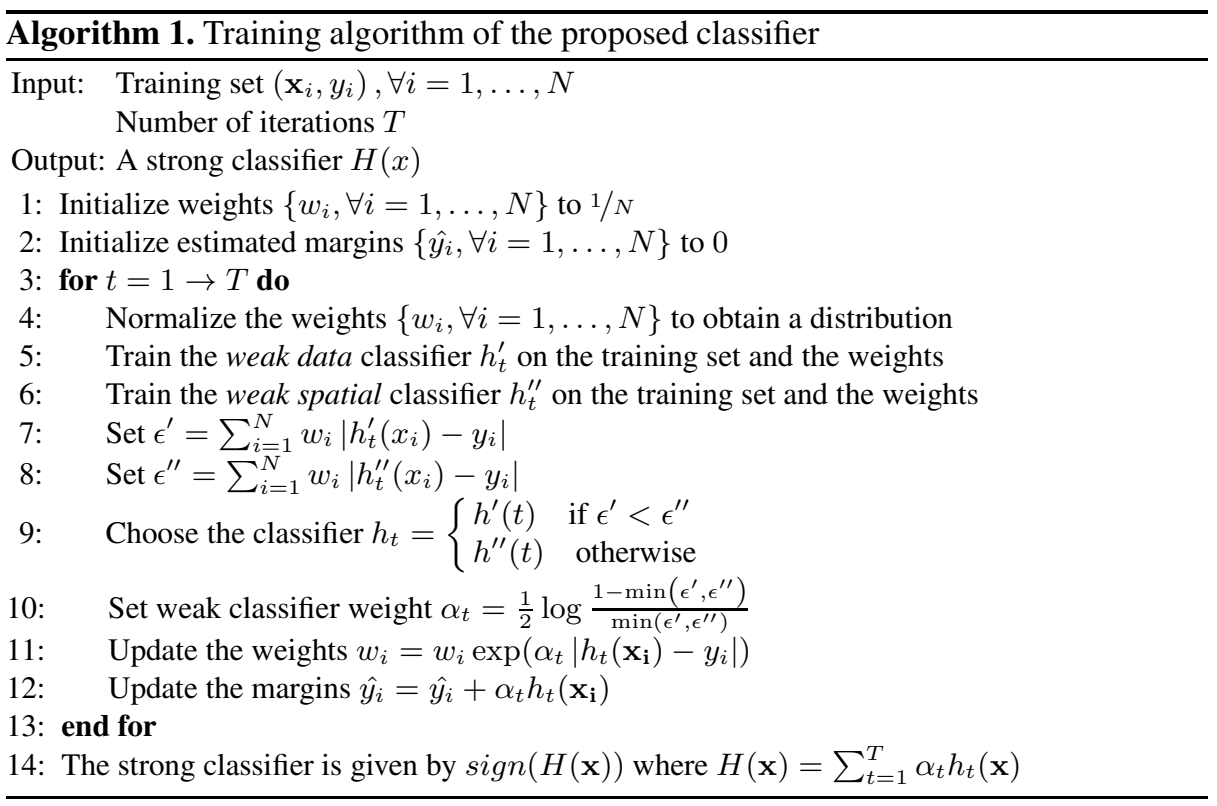

The sum of the weights of positive and negative samples have been scaled to be equal to 0.5 so as to prevent bias to the negative samples if the area of the mass is smaller than that of the background.

\section{Experimental Results}

The method has been tested on a set of images extracted from a publicly available database, the Digital Database for Screening Mammography (DDSM) from the University of South Florida [6]. The database contains 2620 mammography screening exams, each containing four images corresponding to the cranio-caudal (CC) view and the medio-lateral oblique (MLO) view for each breast. The images were obtained by scanning the film-screen mammograms with a sampling rate varying from 42 to 50 microns and 12 bits per pixel. For our tests we considered images coming from both benign and malignant cases. In the case of masses, each image in the dataset is annotated with a curve that includes the mass, but the curve generally does not provide a faithful contour of the mass and thus cannot be used as a precise ground truth. Its aim is merely to delimit an area surely containing the lesion. For this reason, an internal contour $C_{1}$ for each image used in the tests has been drawn in order to enclose the core of the mass together with an external contour $C_{2}$ beyond which the mass does not extend. The pixels internal to $C_{1}$ provide the samples for the positive class, while the negative class is represented by the pixels placed between $C_{2}$ and a rectangle including $C_{2}$. The size of the rectangle is such that the number of samples in the two classes is balanced. The pixels between $C_{1}$ and $C_{2}$ are the samples to be classified (test set) at the end of the training phase. Fig. 1 shows the various contours on one of the images used in our tests. 


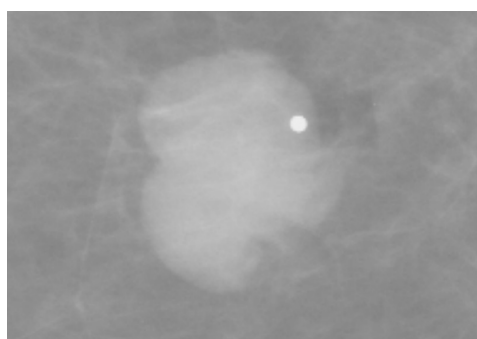

(a)

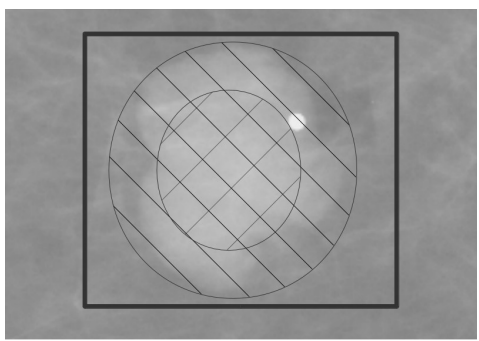

(b)

Fig. 1. An example of the map assumed as input to the classifier. In (a) a region of the mammogram containing a mass and in (b) the map drawn for the ROI. The external rectangle indicates the whole ROI while the smaller ellipse is the core of the mass (positive class). The region between the bigger ellipse and the rectangle is background (negative class) while the region between the two ellipses are the unlabeled pixels.

The features used by the classifiers have been evaluated as described in Sect 2.1, in particular, we have chosen $n=11$ for the size of the window which defines the neighborhood of the pixel. The number $T$ of iterations required to build the classifiers described in Sect.2.2 is set to 10 .

In the following figures the segmentation obtained on some images are shown. In particular, Fig. 2 reports the area of the mass found on the image previously shown. The thick contour includes the pixels classified as positive (i.e., belonging to the mass) by the trained classifier. It appears to faithfully follow the border of the mass which, in this case, can be clearly localized.

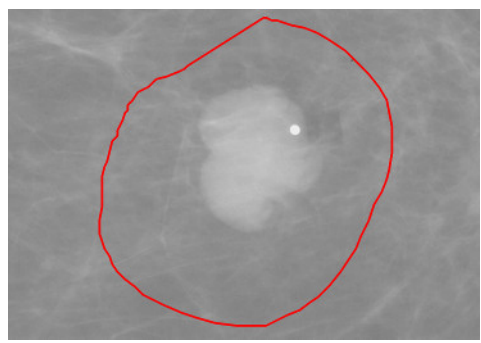

(a)

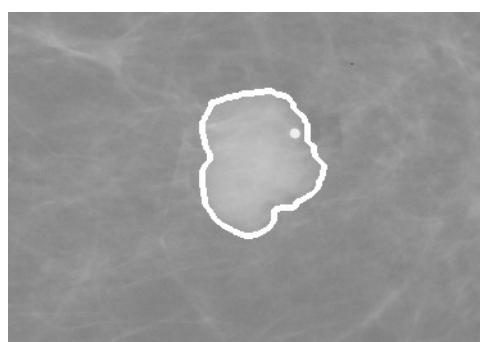

(b)

Fig. 2. (a) The ground truth of the image C_0082_LCC; (b) the boundary obtained through the proposed approach

Nevertheless the method provides a good output also in more difficult cases. For example, Figs. 3, 6 show the results obtained on masses which cannot be easily distinguished from the tissue surrounding them.

The method has been tested also on spiculated masses, i.e., masses with thin and elongated pieces of tissue extending from their perimeter. Some results are shown in 


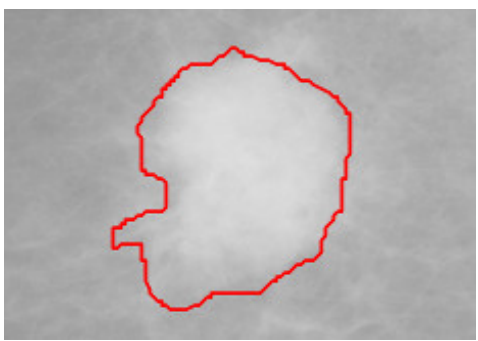

(a)

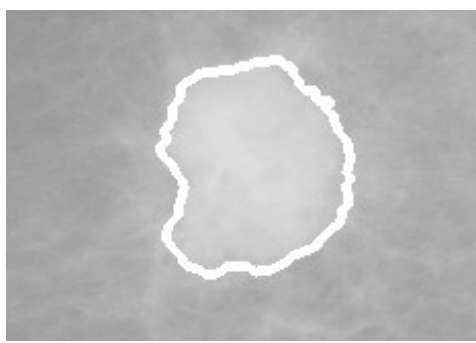

(b)

Fig. 3. (a) The ground truth of the image B_3084_RCC; (b) the boundary obtained through the proposed approach

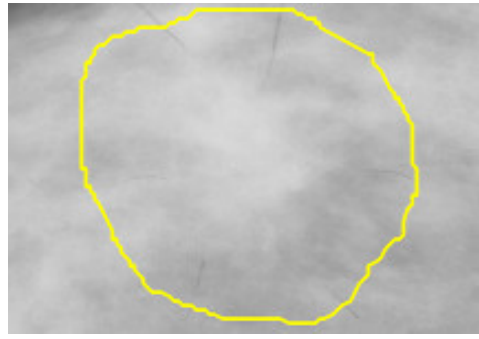

(a)

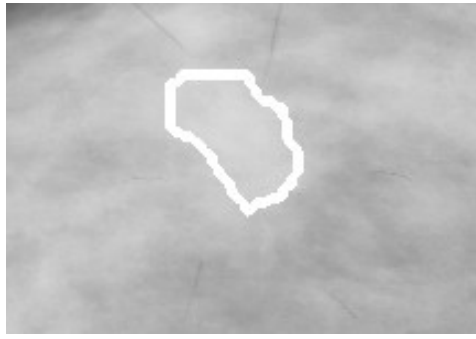

(b)

Fig. 4. (a) The ground truth of the image C_0041_RCC; (b) the boundary obtained through the proposed approach

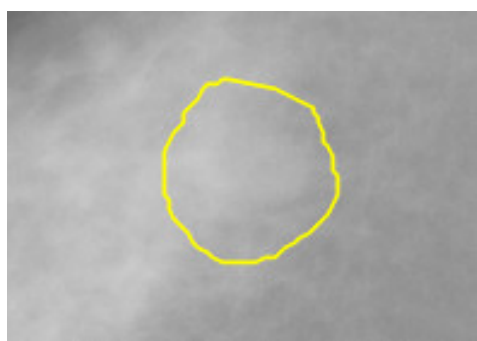

(a)

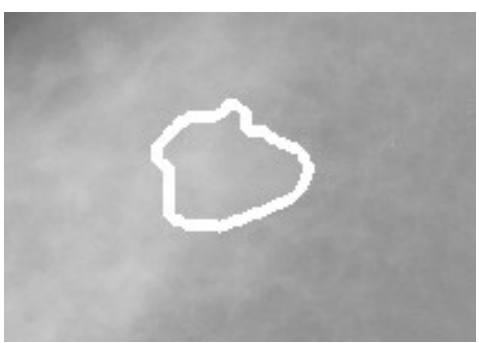

(b)

Fig. 5. (a) The ground truth of the image B_3142_RCC; (b) the boundary obtained through the proposed approach 


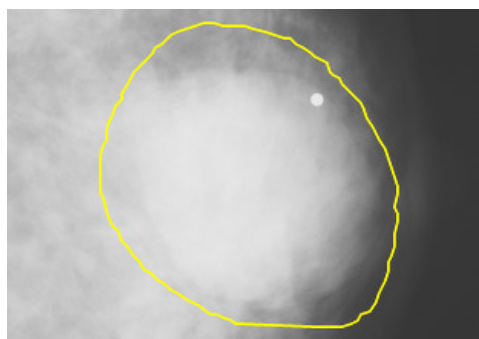

(a)

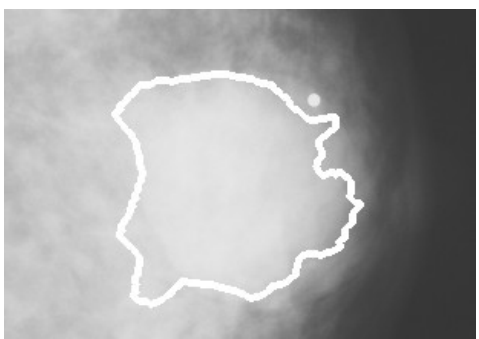

(b)

Fig. 6. (a) The ground truth of the image C_0248_LCC; (b) the boundary obtained through the proposed approach

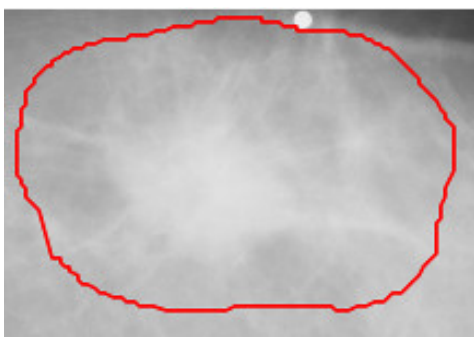

(a)

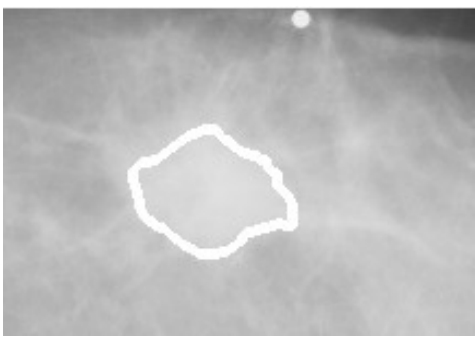

(b)

Fig. 7. (a) The ground truth of the image C_0069_RCC; (b) the boundary obtained through the proposed approach

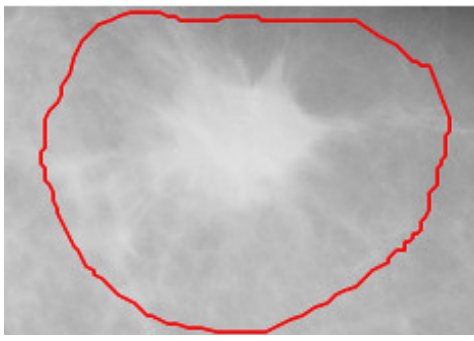

(a)

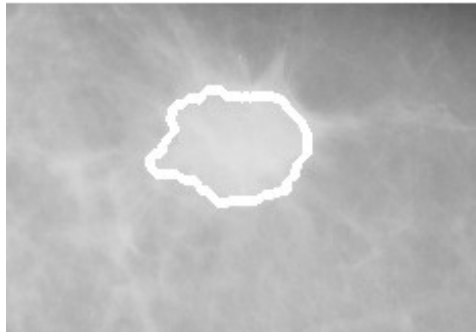

(b)

Fig. 8. (a) The ground truth of the image C_0069_RMLO; (b) the boundary obtained through the proposed approach

Figs. 7 and 8 In these cases the method is able to correctly delineate the central part of the lesion. This is a reasonable behavior because in most cases the shape of the spicules cannot be objectively outlined, while the core of the mass is much more reliably defined.

\section{Conclusions and Future Work}

In this paper we have presented a segmentation method for masses in mammograms that has been inspired by the method proposed in [2]. The first results show that, in 
most cases, such approach provides visually acceptable segmentations. Obviously, a more objective evaluation is still required where this method should be compared with other segmentation approaches, specially considering the differences provided when used in a Computer Aided Detection system. This is the immediate future step in this work. Moreover, we think that some improvements can be obtained specially in defining the training set and in the choice of the features. In particular, a new mechanism should be devised that eliminates the need for two contours and possibly requires only one contour in which the mass is contained. At the same time, texture descriptors should be considered that more suitably discriminate the spatial arrangement of the pixel in the mass from those in the external tissue.

\section{References}

1. Cancer Facts \& Figures 2012. American Cancer Society, Atlanta (2012)

2. Avidan, S.: Spatialboost: Adding spatial reasoning to adaboost. In: Leonardis, A., Bischof, H., Pinz, A. (eds.) ECCV 2006. LNCS, vol. 3954, pp. 386-396. Springer, Heidelberg (2006)

3. Cheng, H., Cai, X., Chen, X., Hu, L., Lou, X.: Computer-aided detection and classification of microcalcification in mammograms: a survey. Pattern Recognition 36, 2967-2991 (2003)

4. Dalal, N., Triggs, B.: Histograms of oriented gradients for human detection. In: CVPR, pp. 886-893 (2005)

5. Giger, M.: Computer-aided diagnosis of breast lesions in medical images. Computing in Science and Engg. 2, 39-45 (2000)

6. Heath, M., Bowyer, K., Kopans, D., Moore, R., Kegelmeyer, W.P.: The digital database for screening mammography. In: Yaffe, M. (ed.) Proc. 5th Int. Workshop on Digital Mammography, pp. 212-218 (2001)

7. Lowe, D.G.: Distinctive image features from scale-invariant keypoints. Int. J. Comput. Vision 60(2), 91-110 (2004)

8. Marrocco, C., Molinara, M., Delia, C., Tortorella, F.: A computer-aided detection system for clustered microcalcifications. Artificial Intelligence in Medicine 50(1), 23-32 (2010)

9. Nishikawa, R.: Current status and future directions of computer-aided diagnosis in mammography. Computerized Medical Imaging and Graphics 31, 1357-1376 (2007)

10. Rangayyan, R., Ayres, F.J., Desautels, J.: A review of computer-aided diagnosis of breast cancer: Toward the detection of subtle signs. Journal of the Franklin Institute 344(3-4), 312-348 (2007)

11. Tang, J., Rangayyan, R., Xu, J., El Naqa, I., Yang, Y.: Computer-aided detection and diagnosis of breast cancer with mammography: recent advances. Trans. Info. Tech. Biomed. 13, 236-251 (2009) 